\title{
Force Characteristics of Trocar Insertion Abdomen in Laparoscopic Surgery
}

\author{
Junpeng Sun, Kotaro Tadano \\ Tokyo Institute of Technology, Laboratory for Future Interdisciplinary \\ R2-418 4259 Nagatsuta-cho, Midori-ku, Yokohama, Kanagawa, Japan \\ junpengsun@hotmail.com
}

\begin{abstract}
Firstly, trocar insertion is an essential step in laparoscopic surgery and the necessary of researching in force characteristics when the trocar inserts abdomens is explained. Secondly, an experimental platform for measuring force when the trocar inserts abdomens is designed. Thirdly, we have developed circuit controlling system to ensure that signal readings are right and motor moves precisely under our control. Then experiments that drives the trocar inserting abdomens and measures force when inserting were conducted. By analysing the force characteristics, conclusions come that the maximal force when the trocar inserts abdomens is about $50 \mathrm{~N}$ and when the force arrives the biggest peak, the trocar has not penetrated out of abdomens yet. The smaller peak next to the maximum force peak is the signal of penetrating-out. We have made an explanation for the force characteristics mentioned above from aspects of insertion principle and abdomen structure. We believe the conclusion offers references for deep research in trocar insertion abdomen and development of trocar insertion robot in the future.
\end{abstract}

Keywords: force characteristics, trocar insertion, laparoscopic surgery, abdomen structure, insertion principle

\section{Introduction}

Trocar insertion is the first step in majority of minimally invasive surgery, where a long sharp tool (which is called trocars) penetrates through the abdominal wall of patients. It makes necessary ports to insert the laparoscopic and endoscopic tools for operating. However, trocar insertion has been extensively cited for causing surgical injuries (most patient injuries appear to occur during the initial insertion of the trocar) because it is done primarily only with operators' haptic sensation and there is considerably little or no visual feedback during inserting [1-3].

To avoid injuries to patients' viscera and artery caused by operators' error, some researches have been done about trocar insertion. To get the visual feedback to remind operators of forcing and stopping the trocar accurately, some operators put the endoscopy into the trocar to create an optical trocar [4-5] as it is shown in Fig 1. To make operators master the key-skill of the trocar insertion procedure, researchers have developed a VR training system for trocar insertion [6-7]. On the other hand, master-slave assisted-robots for perfecting the operation of surgeons have been developed by Ashiwad Chowriappa [8].

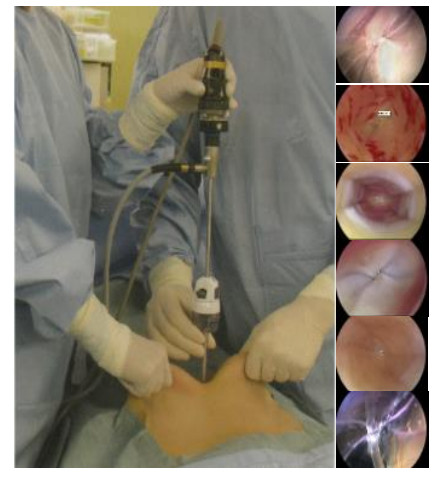

Fig. 1: Insertion with an optical trocar. 
However, researches mentioned above did not analyse the force characteristics when trocar inserts abdomen. And we believe that the force characteristics are important for deep research and development of automatic trocar insertion robot in the future. Thus, this paper focuses on analysis of force characteristics when trocar inserts abdomens. We have had some inserting experiments and got some conclusions. In addition, we explained the force characteristics reasonably with abdomen structure and insertion principle.

In this paper, section 2 tells design of the experimental platform, which includes selection of components and mechanical structure. In section 3 we had experiments using streaky pork and got the force characteristics when trocar inserted abdomens. In section 4, we have made a discussion for the force characteristic mentioned above from aspects of insertion principle and abdomen structure. Finally, conclusions come that the maximal force when trocar inserts abdomen is about $50 \mathrm{~N}$ and when the force arrives peak, trocar has not penetrated out of abdomens yet. The smaller peak next to the maximum force peak is the signal of penetrating-out, which is caused by tissue structure of abdominal wall.

\section{Design of experimental platform}

Table1. Components of experimental platform

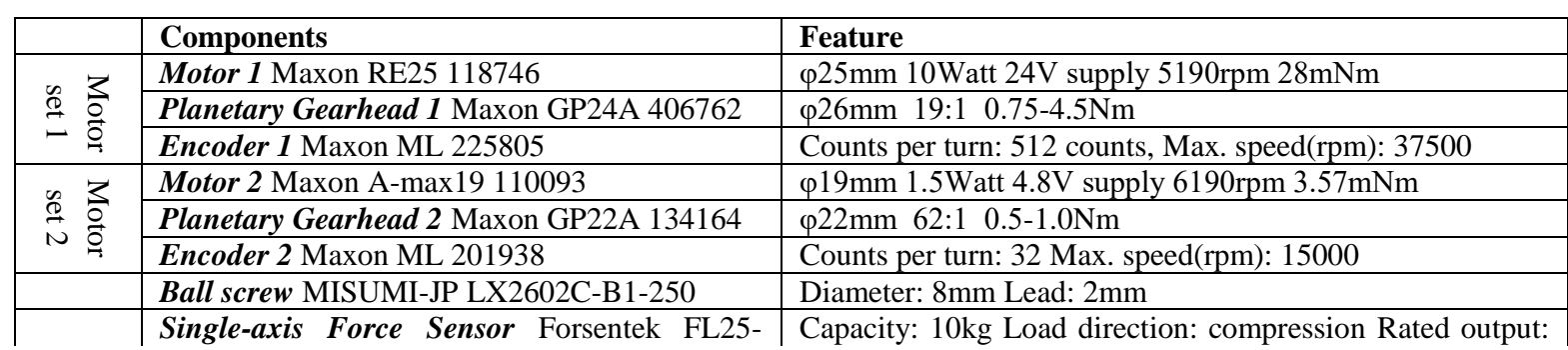

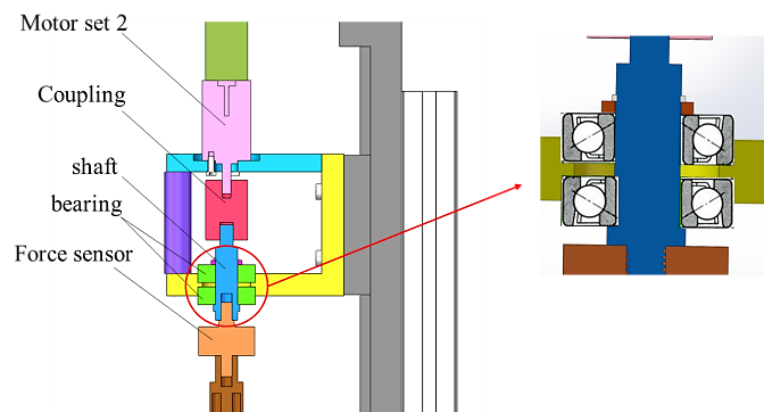

Fig. 2: Mechanical design.
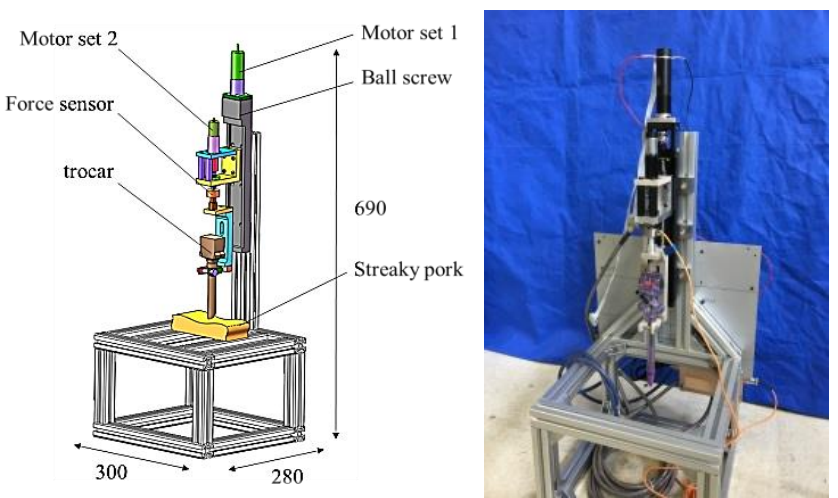

Fig. 3: Design of the experimental platform and the prototype. 
Mechanical design of the experimental platform is improved repeatedly. As is shown in Fig.2, to bear the vertical force when trocar inserts abdomens, two angular-bearings are maintained in platform and the shaft is jointed with the shaft of Motor set 2 by coupling. By this design, the shaft of motor set 2 does not bear great force in vertical direction but can drive the trocar rotating.

The experimental platform is built as is shown in Fig.3, which has two degrees of freedom (DOF), vertical movement and rotation. The vertical movement is driven by motor 1 and a ball screw, the vertical rotation is driven by motor 2 . To measure the force when trocar inserts abdomens, a single-axis force sensor is maintained above the trocar. The trocar is driven to move downward as it is rotating back and forth.

Components of the experimental platform are listed in Table 1, which consists of two motor sets, a ball screw, a single-axis force sensor and a trocar. Motor set 1 and the ball screw drive the trocar moving vertically. Based on the features in Table 1, outputs of the Motor set 1 is $217 \mathrm{rpm}, 0.53 \mathrm{Nm}$ and the trocar is driven by $100 \mathrm{~N}, 7.23 \mathrm{~mm} / \mathrm{s}$. On the other hand, Motor set 2 drives the trocar rotating around vertical direction. Outputs of the Motor set 2 are 39rpm, $0.22 \mathrm{Nm}$. In addition, the capacity of the single-axis force sensor is $\pm 10 \mathrm{~kg}$, when compression force is plus.

\section{Insertion experiments}

In section2, we have designed an experimental platform, which consists of motors and force sensor. Then we have built the electric control system and made sure motors are driven precisely. In this section, we have done insertion experiments using streaky pork on the platform.

The material we have chosen in experiments is streaky pork(thickness $=45 \mathrm{~mm}$ ), because its organizational structure is the same to human being's abdomen, which consists of skin, subcutaneous fat, rectus muscle, and peritoneum. In addition, streaky pork's hardness and thickness are close to human beings'. And the trocar we chose is a spring trocar (diameter = $12 \mathrm{~mm}$ ), which is the most common in laparoscopic surgery.

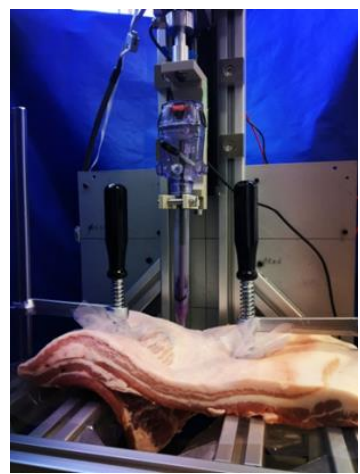

Fig. 4: Insertion experiments using streaky pork.

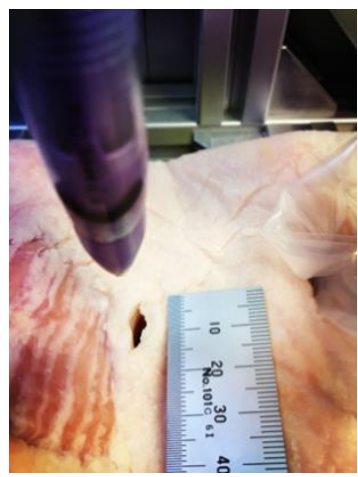

Fig. 5: Insertion wound 


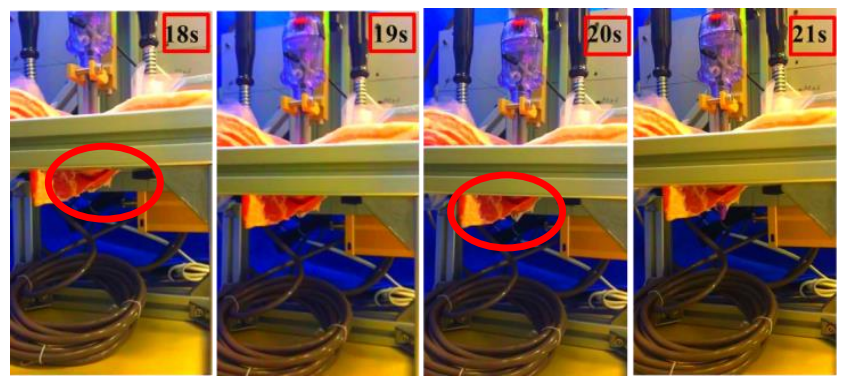

Fig. 6: Process of insertion by pictures in seconds.

Then we drove the trocar moving down in a constant speed using PID position control. We set a first order linear signal to referential position signal, so that we got the constant speed. Normally, surgeons finish the trocar penetrating through abdomens in 20-30s, so we have chosen suitable inserting speed, which is about $4.0 \mathrm{~mm} / \mathrm{s}$, to imitate this work in experimental platform as far as possible. Experiments were executed as shown in Fig.4 and Fig.5. The trocar had moved in a constant speed, $4 \mathrm{~mm} / \mathrm{s}$. And the force measured is shown in Fig.7. In order to analyse force characteristics when trocar insertion abdomen, we have set a high-definition camera (12 million pixels) to record the whole process of insertion as a video and separated the video into pictures by every second, as is shown in Fig. 6.

\section{Discussion}

In Fig.7 we can learn that the maximal force in the whole insertion process is about $42 \mathrm{~N}$. And comparing pictures in Fig.6 with Fig.7, we can see that when the force arrives at peak it is 18th sec in Fig.7, but in this time the trocar had not penetrated out of abdomens yet, which can be learned in Fig.6. And in the pictures of Fig.6 we can see the trocar penetrated out of abdomens in 20th sec. In Fig.7, there is a little force rise in 20th sec. So we considered that the smaller peak next to the maximum force peak is the signal of penetrating-out.

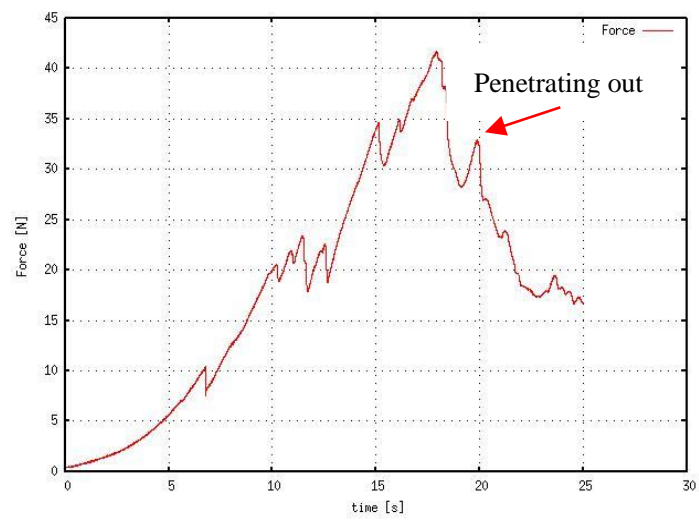

Fig. 7: Force - Time.

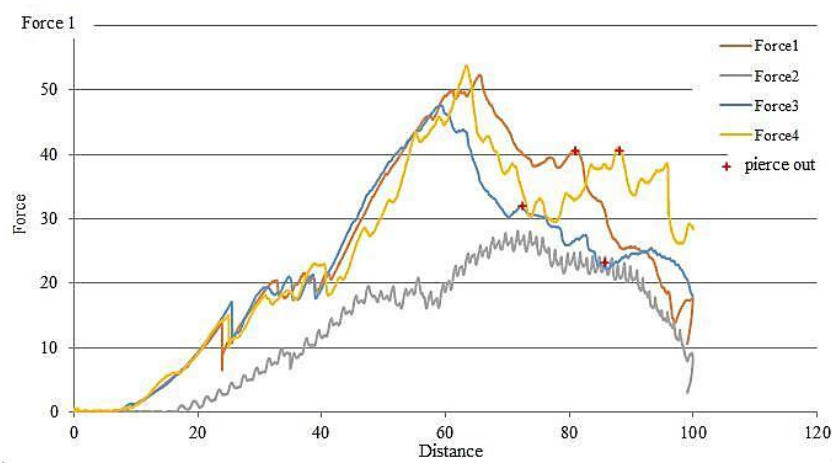

Fig. 8: Force - Distance in different conditions. 
This force characteristic is determined by insertion principle and the abdomen structure. Firstly, when the trocar inserts a kind of tissue, the bearing stress of the tissue grows along with inserting force's growth. When the bearing stress grows to the limited stress, which depends only on the tissue itself, the trocar penetrates out of the tissue. That is insertion principle. Secondly, abdomen consists of skin, subcutaneous fat, rectus muscle, and peritoneum. As is known to all, the firmest tissue of them is rectus muscle. Therefore, when the inserting-force arrives max, bearing stress of the muscle arrives to muscle's limited stress and the trocar penetrates out of the muscle. After that, trocar comes into contact on peritoneum, which is softer than muscle, and the force falls. Then the trocar goes on inserting and the peritoneum deforms. Inserting force rises back along with bearing stress of peritoneum increasing caused by deforming. When bearing stress of peritoneum grows to its limited stress, trocar penetrated out of it and the force falls. The trend of force mentioned above can be seen in Fig.7. As peritoneum is the last layer of abdomen, the smaller peak next to the maximum force peak, which caused by peritoneum-inserting can been considered as the signal of abdomen penetrating-out.

In order to analyse the force characteristics roundly, we have done experiments further. We have set different driving models [Force1(insertion speed: $2 \mathrm{~mm} / \mathrm{s}$, without rotation), Force2(insertion speed: $2 \mathrm{~mm} / \mathrm{s}$, with rotation), Force3(insertion speed: $8 \mathrm{~mm} / \mathrm{s}$, without rotation), Force4(insertion speed: $8 \mathrm{~mm} / \mathrm{s}$, with rotation)]. In conditions above, we have measured forces and got relationships between force and distance trocar moved down, as is shown in Fig.8.

The rotation in this experiment is set to a sine movement which frequency is $1 \mathrm{~Hz}$ and amplitude is $\pi / 4$. So when there is rotation, the trocar inserts down when and rotates itself.

Comparing the curves of Force1 and Force2, we can learn that Force2 is smaller than Force1 at the same distance. In other words, when inserting speed is $2 \mathrm{~mm} / \mathrm{s}$, insertion with rotation can make fewer injuries than without rotation.

Comparting Force1 and Force3, we can learn that there is not obvious difference between the forces at same distance when it is inserting. So when there is no rotation, there is no obvious difference about the force whether inserting speed is high or not.

Comparing Force2 and Force4, we can learn that inserting with rotation can make fewer injuries in low inserting speed, but cannot make out in high inserting speed.

In addition, we have marked positions with red point when the trocar penetrates out of abdomen, as is shown in Fig.8. We can learn that when trocar penetrates out of abdomen, force has passed the maximal peak and there is a little force rise. Therefore, the conclusion is validated by experiments that the smaller peak next to the maximum force peak is the signal of penetrating-out.

Finally, we have compared the maximal force in every condition, and learned that the maximal force is about $50 \mathrm{~N}$ expect trocar insert in $2 \mathrm{~mm} / \mathrm{s}$ with rotation. Inserting in $2 \mathrm{~mm} / \mathrm{s}$ with rotation is better model because it cases fewer injuries but wastes more time.

\section{Conclusions}

From the discussion mentioned above, we have got conclusions as follow:

1 The estimation method of the moment of trocar penetration on abdomen with force-time curve was studied. The smaller peak next to the maximum force peak is the signal of penetrating-out.

2 When inserting speed is low $(2 \mathrm{~mm} / \mathrm{s})$, insertion with rotation can make fewer injuries than without rotation, but not in high inserting speed $(8 \mathrm{~mm} / \mathrm{s})$.

3 When there is no rotation, no difference is observed in the force-distance curves even with different inserting speeds.

\section{References}

[1] H. T. Sharp, M. K. Dodson, M. L. Draper, et al, "Complications associated with optical-access laparoscopic trocar," Obstet Gynecol., vol. 99, no. 4, pp. 553-5, 2002.

[2] S. Krishnakumar, P. Tambe, "Entry complications in laparoscopic surgery," J Gynec Endoso Surg, vol. 1, pp. 4-11, 2009.

[3] O. Rocco, P. Pietro, L. Flavio, "Needle and trocar injuries in diagnostic laparoscopic under local anesthesia: what is the true incidence of these complications?," Journal of Laparoscopic \& Advanced Surgical Techniques, vol. 13, no. 3, pp. 181-184, doi:10.1089/109264203766207708, 2003.

[4] J. A. Brown, D. Canal, C. P Sundaram, "Optical-access visual obturator trocar entry into desufflated abdomen during laparoscopic; assessment after 96 cases," Endourol., vol. 19, no. 7, pp. 853-5, 2005. 
[5] B. R. Berch, A. Torquati, R. E. Ltfi, et al, "Experience with the optical access trocar for safe and rapid entry in the performance of laparoscopic gastric bypass," Surg Endsc, vol. 20, pp. 1238-41, 2006.

[6] M. Sakaguchi, A. Hayashi, H. Fujimoto, et al, "Development of virtual trocar insertion training system for endoscopic surgery," Int. J. Computer Assisted Radiology and Surgery, pp.159-161, 2006.

[7] M. Sakaguchi, H. Hidaka, J. Arata, et al, "Measurement of Trocar Inserting Motion for Endoscopic Surgery and Development of Force Feedback Device for Training," The 5th Int. Conf. on Ubiquitous Robots and Ambient Intelligence, pp. 643-646, 2008.

[8] A. Chowriappa, R. Wirz, Y. W. Seo, A. Reddy, T. Kesavadas, P. Scott, K. Guru, T. Kesavadas, “A Predictive Model for Haptic Assistance in Robot Assisted Trocar Insertion," IEEE World Haptics Conference 2013, 978-1-4799-0088$6 / 13$. 